\title{
Thermomagnetic convection in a cylindrical enclosure. Part 1: Zero gravity
}

\author{
Mikhail S. Krakov ${ }^{1, *}$, Ivan V. Nikiforov ${ }^{2}$ \\ ${ }^{1}$ Belarusian National Technical University, 65 Independence Ave., 220013 Minsk, Republic of Belarus \\ ${ }^{2}$ Belarusian State University, 4 Independence Sq., 220050 Minsk, Republic of Belarus
}

\begin{abstract}
The effects of a uniform external magnetic field on the thermomagnetic convection of magnetic fluid in a cylindrical enclosure are numerically studied. It was found that uniform magnetic field can be cause the convection in the non-uniformly heated magnetic fluid in a cylindrical enclosure even in the case of zero gravity. There are two threshold values of fluid magnetization and temperature gradient. Above the first threshold the convective flow is realized as circular flow, above the second one there are two cells in the enclosure. The angle between a magnetic field strength and a temperature gradient is found to be significant factor influencing structure of a convective flow and heat transfer control.
\end{abstract}

\section{Introduction}

Natural convection of magnetic fluids has attracted much research over the years $[1-6]$. The additional body force $\mu_{0} M \nabla H$ gives an opportunity to control the natural convection under magnetic field. This ponderomotive Kelvin force drives the stronger magnetized colder fluid to the regions with a stronger magnetic field. Such a motion is referred to as thermomagnetic convection. The thermomagnetic convection has a great potential for many engineering applications, such as electronic cooling devices, heat exchanger, and pure magnetic convection in space engineering.

In the case of uniform magnetic field the Kelvin force is equal to zero and magnetic field can not to effect convection. But there are two factors which even in the case of uniform external magnetic field give an opportunity to control the thermoconvection in an enclosure.

The first one has been founded by Finlayson [7] and studied in details in [4, 8] for infinite flat layers. It is shown that uniform transversal external magnetic field becomes nonuniform in the infinite layer of a heated magnetic fluid and influence a convection. Magnetogravitational convection in a vertical layer of magnetic fluid in an oblique magnetic field is studied in [9].

The second factor is the geometry of a magnetic fluid-filled enclosure. Any finite enclosure such as square or cube has the corners and magnetic field becomes nonuniform near them because of boundary conditions for magnetic field. The effect of the external uniform magnetic field on the thermomagnetic convection in square and cubic enclosures was studied in [10-13].

It is interesting to exclude the factor of geometry and study the convection in the situation when the external magnetic field remains uniform in an enclosure. Such enclosure with finite dimension would be very convenient both for theoretical and experimental investigation.

It's well known that uniform external magnetic field remains to be uniform in the magnetic enclosures formed by the surfaces of the second order: the cylinder magnetized across the axes, ellipsoid or sphere. Experimental study of the thermomagnetic convection in the sphere is presented in [14]. We study the effect of the uniform external magnetic field on 2D-convection in the cylindrical enclosure in order to understand the results of [14] too.

\section{Physical model}

Cylindrical enclosure heated from below is one of the classical and the most considered configuration for the study of natural convection because of its relative simplicity and practical importance. Fig. 1 shows the schematic view of the physical model considered in this paper. The cylindrical enclosure with the radius $R$ filled with magnetic fluid is surrounded with the solid massive with the radius $R_{\infty}$. The temperature at the external boundary is fixed as

$$
T=-\frac{T_{b}-T_{t}}{2 R} y+\frac{T_{b}+T_{t}}{2}
$$

so, the temperature gradient at the "infinity" is oriented vertically downwards and equal to

$$
\gamma_{0}=-\frac{T_{b}-T_{t}}{2 R}
$$

If the thermal conductivity of the fluid and the solid massive are equal then $T_{\mathrm{b}}$ is the temperature at the bottom point of the cylinder $(x=0, y=-R)$ and $T_{\mathrm{t}}-$ at the top one, otherwise the temperature gradient for the regime of conductivity is constant and equal to [16] 


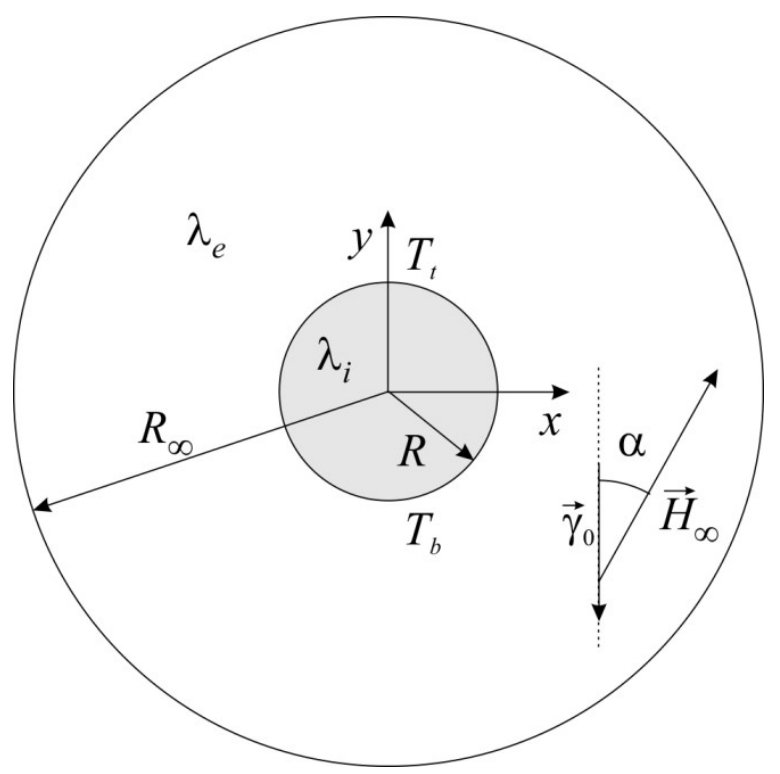

Fig. 1. Problem geometry.

$$
\gamma=\frac{2 \gamma_{0}}{1+\lambda_{i} / \lambda_{\mathrm{e}}}
$$

The magnetic field is supposed to be uniform at the "infinity" $R_{\infty}: \mathbf{H}_{\infty}=\left\{H_{\infty x}, H_{\infty y}\right\}$. If the magnetic permeability of the fluid is $\mu$ and the solid massive is nonmagnetic than, in the isothermal case, the magnetic field in the cylinder is uniform and equal to [17]

$$
\mathbf{H}=\frac{2 \mathbf{H}_{\infty}}{1+\mu(H) / \mu_{0}}
$$

where $\mu_{0}$ is the permeability of vacuum.

In our computations we use the properties of magnetic fluid from the experimental paper [14]:

Density, $\rho$ $1370 \mathrm{~kg} / \mathrm{m}^{3}$

Dynamic viscosity, $\eta$

$0.069 \mathrm{~Pa} \cdot \mathrm{s}$

Kinematic viscosity $v=\eta / \rho$

Thermal conductivity, $\lambda_{i}$ $5.04 \cdot 10^{-5} \mathrm{~m}^{2} / \mathrm{s}$

Magnetization saturation, $M_{\mathrm{S}}$ $0.15 \mathrm{~W} /(\mathrm{m} \cdot \mathrm{K})$ $44.9 \mathrm{kA} / \mathrm{m}$

The heat capacity of the magnetic fluid could be calculated taking into account the additivity law. As the heat capacities of the surfactant (oleic acid) and the carrier fluid (transformer oil) are very close and equal to $c_{\mathrm{p}}^{\text {fluid }}=1.75 \mathrm{~kJ} /(\mathrm{kg} \cdot \mathrm{K})$ then the heat capacity of the magnetic fluid can be calculated as

$$
c_{\mathrm{p}}=c_{\mathrm{p}}^{\text {solid }} \varphi_{\mathrm{s}}+c_{\mathrm{p}}^{\text {fluid }} \varphi_{\mathrm{f}}
$$

where $\varphi_{\mathrm{s}}$ is the volume concentration of the solid phase and $\varphi_{\mathrm{f}}$ is the volume concentration of liquid phase (oleic acid and transformer oil). The volume concentration of solid phase can be calculated through the density of magnetic fluid and its components. As for transformer oil $\rho_{\mathrm{f}}=817 \mathrm{~kg} / \mathrm{m}^{3}$ and for magnetite $\rho_{\mathrm{s}}=5000 \mathrm{~kg} / \mathrm{m}^{3}$, the volume concentration of the solid phase can be estimated as

$$
\varphi_{s}=\frac{\rho-\rho_{f}}{\rho_{s}-\rho_{f}}=0.132
$$

Heat capacity of the magnetite is $c_{\mathrm{p}}^{\text {solid }}=0.52$ $\mathrm{kJ} /(\mathrm{kg} \cdot \mathrm{K})$ and in according to (5) the heat capacity of the magnetic fluid is $c_{\mathrm{p}}=1.58 \mathrm{~kJ} /(\mathrm{kg} \cdot \mathrm{K})$. Then the thermal diffusivity is $\kappa_{\mathrm{mf}}=\lambda_{l} / \rho c_{\mathrm{p}}=6.9 \cdot 10^{-8} \mathrm{~m}^{2} / \mathrm{s}$.

We assume, as in [14], that the cylindrical enclosure is surrounded by Plexiglas massive with the properties: $\rho=1190 \mathrm{~kg} / \mathrm{m}^{3}, \lambda=0.18 \mathrm{~W} /(\mathrm{m} \cdot \mathrm{K}), c_{\mathrm{p}}=1.27 \mathrm{~kJ} /(\mathrm{kg} \cdot \mathrm{K})$, and thermal diffusivity $\kappa_{\mathrm{e}}=1.2 \cdot 10^{-7} \mathrm{~m}^{2} / \mathrm{s}$.

The dependence of the magnetization of a magnetic fluid with monodisperse particles on a magnetic field is described by the well-known Langevin function. But real magnetic fluids contain particles with the different sizes and this dependence is much more complicated. So, we use empiric relationship which rather correctly describes the experimental dependences of the magnetization on the magnetic field [15]

$$
M(H)=M_{\mathrm{S}} \frac{\chi_{0} H / M_{\mathrm{S}}}{1+\chi_{0} H / M_{\mathrm{S}}}=M_{\mathrm{S}} \frac{\chi_{0} \widehat{H}}{1+\chi_{0} \widehat{H}}
$$

Here $\chi_{0}$ is the initial magnetic susceptibility, the dimensionless value marked by "^^", $\widehat{H}=H / M_{\mathrm{S}}$. In futher computations we used $\chi_{0}=2$ which corresponds to real magnetic fluids. Taking into account equation (7), the equation (4) has an analytical solution: the magnetic field in the cylindrical enclosure filled with a magnetic fluid is related with the external uniform magnetic field by the equation

$$
\widehat{H}=\frac{\left(2 \chi_{0} \widehat{H}_{\infty}-2-\chi_{0}\right)+\sqrt{\left(2 \chi_{0} \widehat{H}_{\infty}-2-\chi_{0}\right)^{2}+16 \chi_{0} \widehat{H}_{\infty}}}{4 \chi_{0}}
$$

\section{Governing equations}

\subsection{Momentum equation}

The momentum equation including gravity buoyancy force and magnetizing force is as follows [3]

$$
\begin{gathered}
\rho\left[\frac{\partial \mathbf{u}}{\partial t}+(\mathbf{u} \cdot \nabla) \mathbf{u}\right]=-\nabla p+\eta \Delta \mathbf{u}+\rho \mathbf{g}+\mu_{0} M \nabla H . \\
\text { The fluid is assumed to be an incompressible fluid } \\
\operatorname{div} \mathbf{u}=0 .
\end{gathered}
$$

For the thermal convection with low temperature difference, the classical Boussinesq approximation that the thermophysical properties of the fluid are assumed to be constant except for the density and the magnetization difference for buoyancy force is used.

There are two reasons for dependence of a fluid magnetization on the temperature: change of the particles magnetic moment and thermal expansion of the fluid [4]. As the Curie temperature for the particles of magnetite is $858 \mathrm{~K}$, around a reference state $T_{0}$ change of the magnetic moment is negligible and, if magnetic field is not very small, only thermal expansion is the reason of magnetization change. Then we can write [4]

$$
\left(\frac{\partial M}{\partial T}\right)_{0}=-M_{0} \beta_{m f}=-M_{0}\left[\varphi_{s} \beta_{s}+\left(1-\varphi_{s}\right) \beta_{f}\right]
$$

where $M_{0}$ is determined with Eq. (7), $\beta_{s}$ is the coefficient of thermal expansion of solid particles, and $\beta_{f}$ - of fluid. As for magnetite $\beta_{s}=2 \cdot 10^{-4} \mathrm{~K}^{-1}$, and for transformer oil $\beta_{f}=6.9 \cdot 10^{-4} \mathrm{~K}^{-1}, \beta_{m f}=6.25$. $10^{-4} \mathrm{~K}^{-1}$.

Using Eq. (7) $(\partial M / \partial H)_{0}$ can be written as

$$
\left(\frac{\partial M}{\partial H}\right)_{0}=\frac{\chi_{0}}{\left(1+\chi_{0} \widehat{H}_{0}\right)^{2}}
$$

Thus, the equation of state finally can be written as

$$
\begin{gathered}
M(H, T)=M_{0}+\frac{\chi_{0}}{\left(1+\chi_{0} \widehat{H}\right)^{2}}\left(H-H_{0}\right)-M_{0} \beta_{m f}\left(T-T_{0}\right) \\
=M_{0}+a(H)\left(H-H_{0}\right)+b(H)\left(T-T_{0}\right)
\end{gathered}
$$

Then the momentum equation can be written as follows: 
$\rho\left[\frac{\partial \mathbf{u}}{\partial t}+(\mathbf{u} \cdot \nabla) \mathbf{u}\right]=-\nabla(p)+\eta \Delta \mathbf{u}-\rho_{0} \beta_{m f}\left(T-T_{0}\right) \mathbf{g}+$ $\mu_{0}\left[\begin{array}{c}a(H)\left(T-T_{0}\right) \nabla H+b(H)\left(H-H_{0}\right) \nabla H \\ +M_{0} \nabla\left(H-H_{0}\right)\end{array}\right]$

We will use a stream function $\psi$ and a vortex $\omega$ as follows:

$$
u_{x}=\frac{\partial \psi}{\partial y}, u_{y}=-\frac{\partial \psi}{\partial x}, \omega=\frac{\partial u_{y}}{\partial x}-\frac{\partial u_{x}}{\partial y}=-\Delta \psi
$$

After an operation curl applied to Eq. (15) and taking into account that $\nabla \times \mathbf{H}=0$, the momentum equation becomes

$$
\begin{aligned}
& \frac{\partial \omega}{\partial t}+(\mathbf{u} \cdot \nabla) \omega=v \Delta \omega+\beta_{m f} g \frac{\partial T}{\partial x}+ \\
& \frac{\mu_{0} M_{S} \beta_{m f}}{\rho} \frac{\chi_{0} \widehat{H}_{0}}{1+\chi_{0} \widehat{H}_{0}}\left(\frac{\partial H}{\partial x} \frac{\partial T}{\partial y}-\frac{\partial H}{\partial y} \frac{\partial T}{\partial x}\right)
\end{aligned}
$$

\subsection{Energy equation}

This equation has a standard form:

$$
\rho c_{p}\left[\frac{\partial T}{\partial t}+(\mathbf{u} \cdot \nabla) T\right]=\lambda \Delta T
$$

\subsection{Magnetic field}

A magnetic field both in the enclosure and in the external space is described with Maxwell's equations for nonconducting fluids

$$
\nabla \times \mathbf{H}=0, \quad \nabla \cdot \mathbf{B}=0, \quad \mathbf{B}=\mu_{0}(\mathbf{H}+\mathbf{M})
$$

As for the magnetic fluids with magnetite particles the vectors of magnetic field strength and magnetization are parallel for flow velocities typical for convection, i.e. $\mathbf{M}=\chi(H, T) \mathbf{H}$, then, using (7), (11) and (12), we get

$$
\begin{gathered}
\mathbf{B}=\mu_{0}(1+\chi(H, T)) \mathbf{H}= \\
\mu_{0}\left(1+\frac{\chi_{0}}{1+\chi_{0} \overparen{H}}\left[1-\beta_{m f}\left(T-T_{0}\right)\right]\right) \mathbf{H}=\mu_{0} \hat{\mu}(H, T) \mathbf{H}
\end{gathered}
$$

As $\nabla \times \mathbf{H}=0$ the magnetic field strength can be expressed as the gradient of a scalar potential $F$

$$
\mathbf{H}=\nabla F
$$

and finally the magnetic field is described with the nonlinear equation

where

$$
\nabla \cdot(\hat{\mu}(|\nabla F|, T) \nabla F)=0
$$

$$
\hat{\mu}(\nabla F, T)=1+\frac{\chi_{0}}{1+\chi_{0}|\nabla F| / M_{S}}\left[1-\beta_{m f}\left(T-T_{0}\right)\right]
$$

\subsection{Boundary conditions}

On the walls of the enclosure, no-slip conditions for the velocity are applied: the stream function is equal to zero on the walls of the enclosure; the vortex is calculated from the Eq. (15 taking into account the curvature of the walls.

The temperature at the "infinity" $R_{\infty}$ is determined by means of the Eq. (1) and the conditions of continuity must be fulfilled at the boundary of the enclosure

$$
T_{i}=T_{e}, \quad \lambda_{i} \frac{\partial T}{\partial n}=\lambda_{e} \frac{\partial T}{\partial n}
$$

The external magnetic field is uniform and at the "infinity" $R_{\infty}$ is equal to $\mathbf{H}_{\infty}=\left\{H_{\mathrm{x} \infty}, H_{\mathrm{y} \infty}\right\}$, i.e. the scalar potential is determined at the external boundary as

$$
F=H_{x \infty} x+H_{y \infty} y
$$

The conditions $\left(\mathbf{B}_{i}-\mathbf{B}_{e}\right) \cdot \mathbf{n},\left(\mathbf{H}_{i}-\mathbf{H}_{e}\right) \cdot \boldsymbol{\tau}=0$ must be fulfilled at the boundary of the enclosure, i.e.

$$
\hat{\mu} \frac{\partial F_{i}}{\partial n}=\frac{\partial F_{e}}{\partial n}, \quad \frac{\partial F_{i}}{\partial \tau}=\frac{\partial F_{e}}{\partial \tau}
$$

where $\mathbf{n}$ is the unit vector normal to the boundary and $\boldsymbol{\tau}$ is the unit vector tangential to the boundary.

\subsection{Dimensionless form of the equations}

We use as the scales: $[t]=R^{2} / v_{i},[x, y]=R,[T]=\gamma_{0} R,[u]$ $=\kappa_{\mathrm{i}} / R,[H]=M_{\mathrm{S}},[F]=M_{\mathrm{S}} R,[\psi]=\kappa_{i},[\omega]=\kappa_{\mathrm{i}} / R^{2}$. The system of equations can be written in dimensionless form as follows:

$$
\begin{gathered}
\nabla \cdot\left(\mu\left(\left|\nabla \widehat{F}_{i}\right|, \theta\right) \nabla \widehat{F}_{i}\right)=0, \quad \Delta \widehat{F}_{e}=0 \\
\frac{\partial \widehat{\omega}}{\partial t}+\frac{1}{\operatorname{Pr}}(\mathbf{u} \cdot \nabla) \widehat{\omega}=\Delta \widehat{\omega}+\operatorname{Ra} \frac{\partial \theta}{\partial x}+ \\
\operatorname{Ra}_{m} \frac{\chi_{0} \widehat{H}_{0}}{1+\chi_{0} \widehat{H}_{0}}\left(\frac{\partial \widehat{H}}{\partial x} \frac{\partial \theta}{\partial y}-\frac{\partial \widehat{H}}{\partial y} \frac{\partial \theta}{\partial x}\right) \\
\Delta \widehat{\psi}=-\widehat{\omega} \\
\operatorname{Pr} \frac{\partial \theta_{i}}{\partial t}+(\mathbf{u} \cdot \nabla) \theta_{i}=\Delta \theta_{i} \\
\operatorname{Pr} \frac{\partial \theta_{e}}{\partial t}=\frac{\kappa_{e}}{\kappa_{i}} \Delta \theta_{e}
\end{gathered}
$$

Here $\hat{F}=F / R M_{S}, \widehat{H}=H / M_{S}, \widehat{\omega}=\omega R^{2} / \kappa_{i}, \widehat{\psi}=\psi / \kappa_{i}$, $\theta=\left(T-T_{0}\right) / \Delta T, \Delta T=2 \gamma_{0} R, \quad$ the magnetic permeability is equal to 1 for external space and is given by (20) for the fluid in the cylinder.

The Rayleigh number, the magnetic Rayleigh number and the Prandtl number are:

$$
\mathrm{Ra}=\frac{g \beta_{m f} \Delta T R^{3}}{v \kappa}, \operatorname{Ra}_{m}=\frac{\mu_{0} M_{S}^{2} \beta_{m f} \Delta T R^{2}}{\rho v \kappa}, \operatorname{Pr}=\frac{v}{\kappa}
$$

\section{Numerical method}

The problem was solved numerically by finite elements method with triangle elements. The linear interpolation functions are used for the magnetic field scalar potential and the stream function

$$
F=A_{F} x+B_{F} y+C_{F}, \quad \psi=A_{\psi} x+B_{\psi} y+C_{\psi}
$$

and the exponential functions [18] for the temperature and the vortex are used:

$$
\begin{gathered}
\omega=A_{\omega} \exp \left[\frac{U_{\mathrm{el}}}{\operatorname{Pr}}\left(X-X_{\mathrm{max}}\right)\right]+B_{\omega} Y+C_{\omega} \\
T=A_{T} \exp \left[U_{\mathrm{el}}\left(X-X_{\text {max }}\right)\right]+B_{T} Y+C_{T}
\end{gathered}
$$

where the coefficients $A, B, C$ are calculated from the function values in the nodes of the triangle elements, a local coordinate system $X, Y$ is oriented at each element so that the axis $X$ directed along the local velocity $\mathbf{U}_{\mathrm{el}}$ in this element.

The vortex at the solid boundary of the enclosure is calculated with taking into account the curvature of the boundary according to the method supposed in [19].

\section{Results and Discussion}

The Rayleigh number is only one governing parameter in the case of the thermo-gravitational convection. In our case there are three independent dimensionless parameters: the numbers $\mathrm{Ra}$ and $\mathrm{Ra}_{\mathrm{m}}$ and the parameter $\beta_{m f} \Delta T$. The last one specifies the magnetic field gradient in the enclosure through the equations (20) and (24). 
In order to exclude the influence of the gravity and to highlight the influence of the uniform external magnetic field on the thermal convection in the pure state we put the Rayleigh number equal to zero $(\mathrm{Ra}=0)$ in this study. As both parameters $\operatorname{Ra}_{\mathrm{m}}$ and $\beta_{m f} \Delta T$ depend on the temperature difference $\Delta T$, we use in the study $\Delta T$ and the magnetization $M_{0}$ as the independent physical parameters. All the further computations are fulfilled for the fluid with properties presented above and corresponding to the magnetic fluid used in the experiments [14]. The dimensionless radius of the external boundary of the calculation domain is $R_{\infty}=11$, the radius of the enclosure, as in the [14], is $8 \mathrm{~mm}$.

The heat flux across the boundary of the cylinder can be characterized by the Nusselt number which was calculated as

$$
\mathrm{Nu}=\frac{1}{\mathrm{Nu}_{0}} \int_{\vartheta_{1}}^{\vartheta_{2}} \frac{\partial \theta}{\partial n} d \vartheta
$$

where $\mathrm{Nu}_{0}$ is the Nusselt number in the case of conductivity regime, $\vartheta_{1}$ and $\vartheta_{2}$ are the angles for which $\partial \theta / \partial n=0$. This integral was calculated both from $\vartheta_{1}$ to $\vartheta_{2}$ and from $\vartheta_{2}$ to $\vartheta_{1}$. The difference between the results was less than $0.01 \%$.

To validate the quality of the numerical results, the problem of convective stability in the field of gravity in a cylindrical enclosure without magnetic field $\left(\mathrm{Ra}_{\mathrm{m}}=0\right)$ is tested and results are compared with the linear theory solution [16]. Table 1 shows the studied grid systems:

Table No 1. Grid parameters.

\begin{tabular}{|l|l|l|l|l|l|}
\hline No of the grid & 1 & 2 & 3 & 4 & 5 \\
\hline $\begin{array}{l}\text { Number of } \\
\text { nodes }\end{array}$ & 9091 & 16201 & 25381 & 36541 & 49771 \\
\hline $\begin{array}{l}\text { Number of } \\
\text { elements }\end{array}$ & 18000 & 32160 & 50400 & 72720 & 99120 \\
\hline
\end{tabular}

Table 2 illustrates the mesh independency analysis. The grid No 4 is used in all computations presented below.
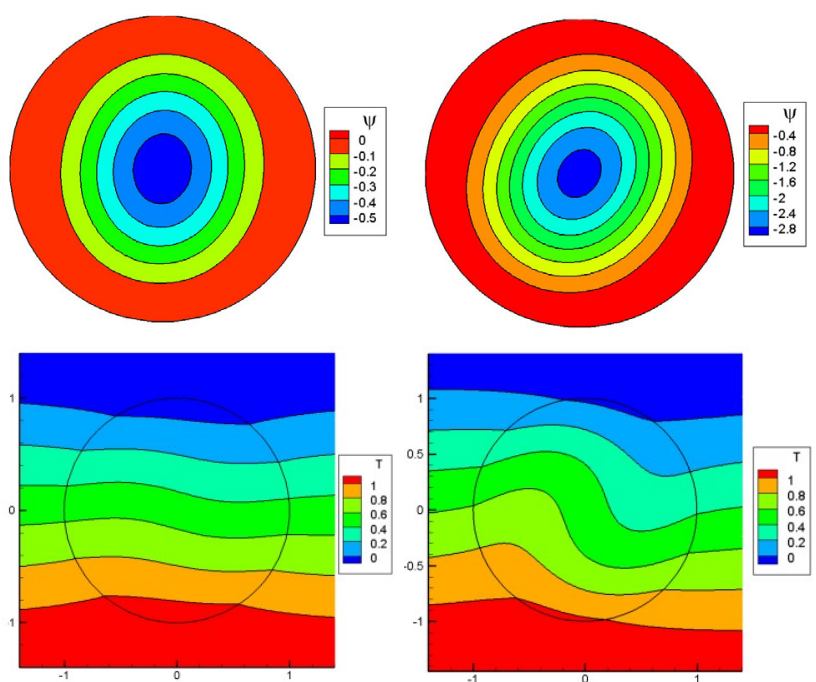

a) $\Delta T=23 \mathrm{~K}$ b) $\Delta T=40 \mathrm{~K}$
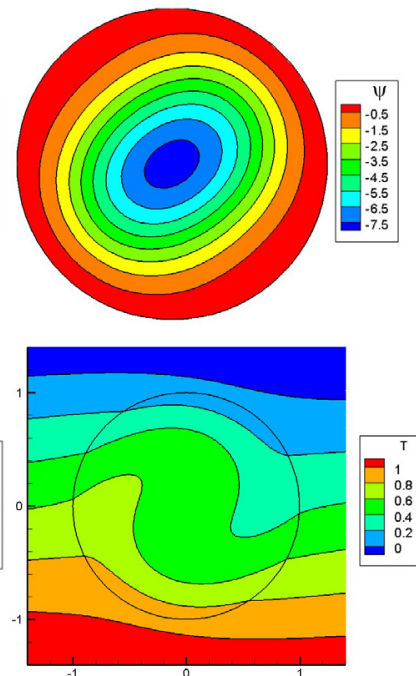

c) $\Delta T=56 \mathrm{~K}$
For the case of zero gravity $(\mathrm{Ra}=0, \mathrm{Ram} \neq 0)$ it was found that there are two types of convective flow

Table 2.

Comparison of the present results of critical Rayleigh number for different grid resolution with linear theory [16].

\begin{tabular}{|l|l|l|l|l|l|l|}
\hline$\lambda_{i} / \lambda_{e}$ & $\begin{array}{l}\text { Galerkin } \\
\text { method } \\
{[16]}\end{array}$ & $\begin{array}{l}\text { Grid } \\
\text { No 1 }\end{array}$ & $\begin{array}{l}\text { Grid } \\
\text { No 2 }\end{array}$ & $\begin{array}{l}\text { Grid } \\
\text { No 3 }\end{array}$ & $\begin{array}{l}\text { Grid } \\
\text { No 4 }\end{array}$ & $\begin{array}{l}\text { Grid } \\
\text { No 5 }\end{array}$ \\
\hline 0.01 & $\mathbf{4 0 8 . 2}$ & 421.52 & 417.63 & 415.32 & 413.8 & 413.01 \\
\hline 0.2 & $\mathbf{3 7 3 . 6}$ & 384.33 & 382.06 & 380.7 & 379.83 & 379.46 \\
\hline 0.5 & $\mathbf{3 7 2 . 3}$ & 379.65 & 378.36 & 377.62 & 377.14 & 377.15 \\
\hline 1 & $\mathbf{4 1 2 . 4}$ & 415.36 & 414.74 & 414.44 & 414.26 & 414.31 \\
\hline 2 & $\mathbf{5 2 7 . 1}$ & 525.88 & 525.97 & 526.11 & 526.30 & 526.40 \\
\hline
\end{tabular}

structures in a cylindrical enclosure (Fig. 2). In contrast to the thermo-gravitational convection, for which the only one structure (a single cell) can be realized, two structures (single cell and two cells) are possible for the thermomagnetic convection in the case of zero gravity.

To check the dependence on the Prandtl number, the problem for the maximum value of the parameters $\left(\mathrm{Ra}=0, \mathrm{Ra}_{\mathrm{m}}=2383686, H_{\infty}=20 M_{\mathrm{S}}, \Delta T=120 \mathrm{~K}\right)$ is solved. Table 3 shows that the results are the Prandtl number almost independent. The value $\mathrm{Pr}=709$ and $\lambda_{i} / \lambda_{e}=0.833$ corresponding to the above-stated properties of the magnetic fluid are used in all computations.

Table 3. The Prandtl number dependency test.
\begin{tabular}{|r|r|r|r|}
\hline \multicolumn{1}{|c|}{$\operatorname{Pr}$} & $\psi_{\min }$ & $\psi_{\max }$ & $\mathrm{Nu}$ \\
\hline 10 & -5.13317 & 5.1306 & 1.6393 \\
\hline 100 & -5.13528 & 5.1358 & 1.6426 \\
\hline 1000 & -5.1356 & 5.1366 & 1.6430 \\
\hline
\end{tabular}

The distribution of the temperature and magnetic field potential in the calculation domain equal to the external boundary conditions and $\psi=0, \omega=0$ are used as initial conditions.

There is no convection for small values of $\Delta T$ and $H_{\infty}$ (remember $\mathrm{Ra}=0$ ). Above some critical values of the fluid magnetization and the temperature difference the circulation flow arises (Fig. 2a). Fig. 3 shows the
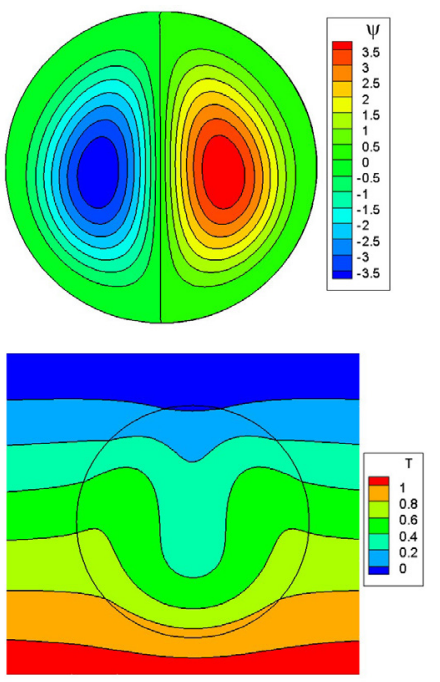

d) $\Delta T=57 \mathrm{~K}$

Fig. 2. Stream function and temperature fields. $\widehat{H}_{\infty}=20, \alpha=0$. correspondence the dependence of the maximum stream 
function on the temperature difference to the square root law of the Landau $\psi \sim\left(\Delta T-\Delta T^{*}\right)^{0.5}$ where $\Delta T^{*}$ is the critical value of the temperature difference. The same

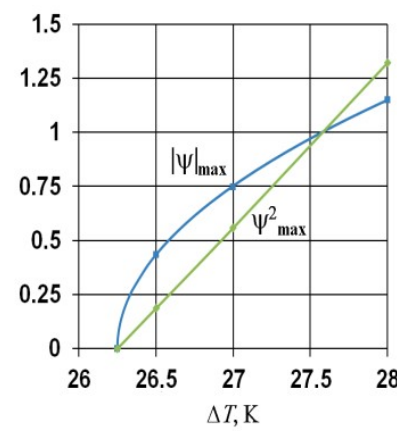

Fig. 3. The maximum of stream function and the square of maximum stream function in the cell vs temperature difference. $\widehat{H}_{\infty}=2, \alpha=0$.

As in the case of thermo-gravitational convection, the circulation flow takes the form of an oval with the increasing of $\Delta T$ and the inclination of the major axis of the oval increases too (Figs. 2b, 2c). The direction of rotation in the case of parallelism of the magnetic field and the temperature gradient is arbitrary and it's possible both clockwise, and counter-clockwise.

The investigations of a linear stability of the basic state in the case of thermo-gravitational convection show [16] that the onset of the convection is possible in one of the four different perturbations: circular flow, two cells oriented vertically and horizontally, and four cells. However, numerous experimental studies and computer simulations found that only one cell flow can be realized. Fig. 2d shows that for sufficient value of the magnetization and the temperature difference two cells flow can be realized for thermo-magnetic convection. Above this second threshold the Nusselt number significantly decreases in comparison with one cell, but the rate of the Nusselt number increasing with the $\Delta T$ growth is much more (Fig. 4).

Fig. 4 shows that, for the given set of the parameters, the convective flow can be realized as two cells if the initial condition is $\psi=0, \omega=0$ and $\Delta T>56 \mathrm{~K}$. But if the initial condition is the one sell solution for $\Delta T<56 \mathrm{~K}$ then the convective flow is one cell for $\Delta T>56 \mathrm{~K}$ too. The corresponding values of the Nusselt numbers are shown with the marker $\square$. It depends on the way of the fluid heating in the enclosure what structure can be realized in the experiment. The circular flow, formed at the small temperature differences, is stable in all range of temperatures if the heating is slow. In the case of quick heating (it corresponds to initial conditions is $\psi=0, \omega=$ 0 and the Prandtl number $\mathrm{Pr}=709$ ) two cell flow is formed for sufficiently high temperature difference. This critical $\Delta T$ depends on the fluid magnetization. Fig. 5 shows two neutral curves which divide the space of $M$ $\Delta T$ on three zones: zone of stable state without convection, zone of the convection with one cell flow character of dependence we can see for the Nusselt number (Fig. 4).

and zone of the two cell flow convection. The connection between the magnetization $M_{0}$ in Fig. 5 and the external magnetic field $H_{\infty}$ is given by the equations (7), (8).

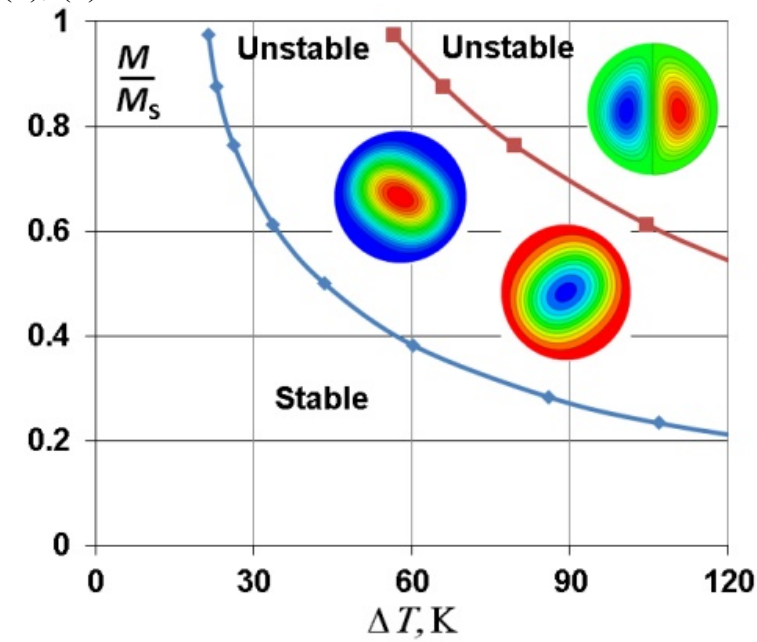

Fig. 5. Neutral curves for $\alpha=0$. The direction of rotation in the case of one cell is arbitrary. $M$ and $\widehat{H}_{\infty}$ are connected through Eqs. (7) and (8).

Till now the magnetic field parallel to the temperature gradient is studied. As the magnetization gradient is formed by the temperature gradient, they are parallel always and, it seems, the direction of the magnetic field vector has not to influence the convective flow. But after the onset of the convection the convective flow distorts the temperature field and, as consequence, distorts the magnetic field too. As a result, the verticality deviation of the magnetic field leads to very complicated interaction with the field of the temperature and can influence the convective flow.

The numerical simulation shows that the maximum absolute value of the stream function for $\Delta T=50 \mathrm{~K}$ (only one cell flow exists for this temperature difference) increases with the increasing of the angle of vertically deviation up to $\pm 30^{\circ}$ and then decreases (Fig. 6a). The dependence of the Nusselt number on the angle of vertically deviation has a similar character (Fig. 6b). The variation of the stream function $\psi$ and the Nusselt number $\mathrm{Nu}$ not very large (less than $2 \%$ ) in this case but it exists. Thus, we can confirm the influence of the uniform magnetic field direction on the convective flow.

For $\Delta T=100 \mathrm{~K}$ and $\alpha=0$ the convective flow has a structure of two cells. In this case the influence of the external magnetic field inclination is more significant (Fig. 7a, b). The flow almost isn't changed in the range of the angles $-28^{\circ} \leq \alpha \leq 31^{\circ}$ : the maximum stream function varies between 6.79 and 6.64 , i.e. within the range of $2 \%$; the Nusselt number varies even less than $\psi:$ in the range of $1.596-1.598$. It should be mentioned that if the angle of the field inclination increases in positive direction then the intensity of the flow in the right cell increases and in the left cell decreases. In the 
case of counter-clockwise inclination of the field we see an opposite situation.

For $76^{\circ}>|\alpha|>31^{\circ}$ the cell with the maximum absolute value of the stream function becomes to dominate and only one cell convective flow is possible in all range of the parameters. The direction of the circulation flow is counter-clockwise for positive $\alpha$ and clockwise for negative angles.

For $90^{\circ}>|\alpha|>76^{\circ}$ we can see two cell structure again.
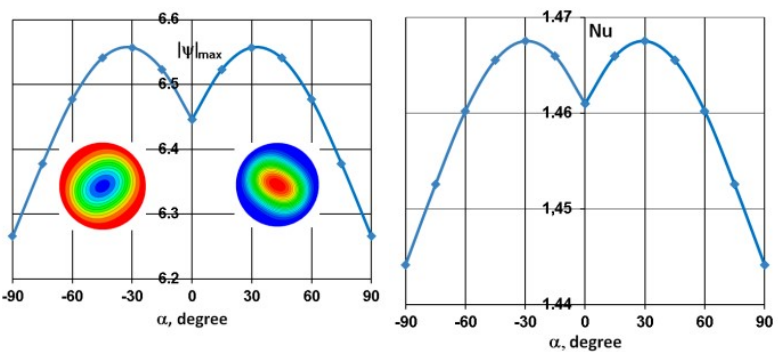

Fig. 6. The effect of magnetic field inclination on maximum stream function (6a) and the Nusselt number (6b). $\Delta T=50 \mathrm{~K}, \widehat{H}_{\infty}=20$.

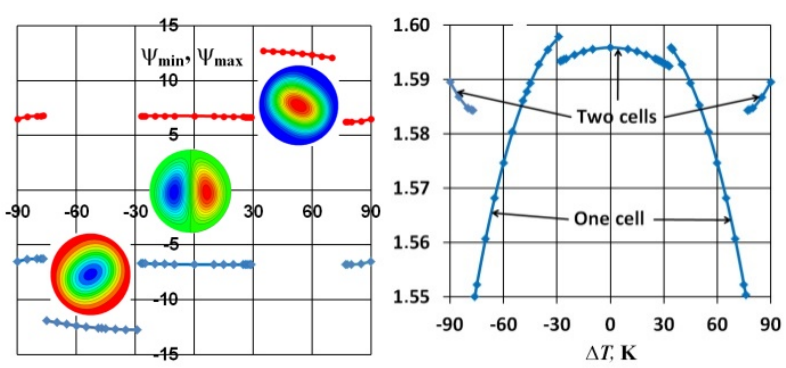

Fig. 7. The effect of magnetic field inclination on maximum stream function (7a) and the Nusselt number (7b). $\Delta T=$ $100 \mathrm{~K}, \widehat{H}_{\infty}=20$.

\section{Conclusions}

The buoyancy-induced thermal convection under external uniform magnetic field is numerically studied in a cylindrical enclosure. The effect of temperature, magnetization, direction of magnetic field and temperature gradient on thermomagnetic convection inside the enclosure is discussed in details. The main important conclusions can be summarized as follows:

1. Convection in a non-uniformly heated cylindrical enclosure can be induced by the external uniform magnetic field even in the case of zero gravity.

2. There are two threshold values of temperature gradient and fluid magnetization.

3. Above the first threshold values the convective flow is realized as circular flow.

4. There are the second threshold values of the parameters of the problem for angles near $0^{\circ}$ and $90^{\circ}$ between magnetic field and temperature gradient. Flow realized above these values is two cells.

5. The direction of rotation in one cell structure is arbitrary for the external magnetic field and temperature gradient parallelism. It is counter- clockwise for positive and clockwise for negative angles.

All these conclusions are right if zero initial conditions for stream function and vortex are used. If the convective structure formed for some parameters (for example, for $\Delta T=100 \mathrm{~K}, M_{0}=0.96, \alpha=0^{\circ}-$ two cells) is used in computer simulation as initial condition for another set of parameters (for example, $\alpha=40^{\circ}$ or $70^{\circ}$ ), restructuring of the convective flow doesn't occur. Thus, it's possible to state that formed structures have some margin of stability.

\section{References}

1. J.R. Carruthers, R. Wolfe, J. Appl. Phys. 39, 5718 5722, (1968)

2. D. Braithwaite, E. Beaugnon, R. Tournier, Nature, 354, 134-136 (1991)

3. R.E. Rosensweig, Ferrohydrodynamics (Cambridge University Press, 1985)

4. V.G. Bashtovoy, B.M. Berkovsky, A. N. Vislovich, Introduction to thermomechanics of magnetic fluids. (Hemisphere, Washington, DC, 1988)

5. B.M. Berkovsky, V.F. Medvedev, M.S. Krakov, Magnetic Fluids: Engineering Applications (Oxford University Press Inc., NY, 1993)

6. E. Blūms, A.O. Cebers, M.M. Maĭorov, Magnetic Fluids (Walter de Gruyter, 1997)

7. B.A. Finlayson, J. of Fluid Mech. 40753 - 767 (1970)

8. V.G.Bashtovoy, J. of Appl. Mech. and Technical Physics. No 3, 126 - 133 (1979) (in Russian.)

9. H. Rahman, S.A. Suslov, J. of Fluid Mech. 795 847-875 (2016)

10. M.S. Krakov, I.V. Nikiforov J. Magn. Magn. Mater. 252 209-211 (2002)

11. M.S. Krakov, I.V. Nikiforov, J. Magn. Magn. Mater. 289 278-280 (2005)

12. S. Kamiyama, M.S. Krakov, I.V. Nikiforov, Magnetohydrodynamics, 40 335-345 (2004)

13. M.S. Krakov, A.G. Reks, I.V. Nikiforov, J. of Magn. Magn. Mater. 289 272-274 (2005)

14. M.T. Krauzina, A.A. Bozhko, P.V. Krauzin, S.A. Suslov. J. of Magn. Magn. Mater. 431241 - 244 (2017)

15. A.N. Vislovich, Magnetohydrodynamics 26178 183 (1990)

16. G.Z. Gershuni, E.M. Zhukhovitskii. Convective Stability of Incompressible Fluids. (I.P.S.T. 1976)

17. L.D.Landau and L.P.Pitaevskii. Electrodynamics of Continuous Media, Second Edition: Volume 8 (Course of Theoretical Physics S). (ButterworthHeinemann 1984)

18. S. Patankar, Numerical Heat Transfer and Fluid Flow. Hemisphere Series on Computational Methods in Mechanics and Thermal Science (Taylor and Fransis, London, 1980).

19. M.S. Krakov, Numer. Heat Transfer, Part B Fundamentals 21/2 125 - 145 (1992) 